\title{
CD23 bridges the gap
}

The structure of the low-affinity human immunoglobulin $E(\lg E)$ receptor $C D 23$, revealed on page 751, accounts for its provocative behavior. Hibbert and colleagues show that soluble CD23 (sCD23) can simultaneously bind IgE and the complement receptor $C D 21$ on $B$ cells. This binding dexterity helps explain how $C D 23$ and $\lg E$ cooperate to ramp up the synthesis of more $\lg \mathrm{E}$.

The production of $\mathrm{IgE}$-the signature antibody of allergic diseases-is enhanced when sCD23 (which is cleaved from the surface of activated B cells by endogenous or pathogen-derived proteases) binds to CD21 on other B cells. But it was not clear why sCD23 binding to CD21 enhanced the production of IgE but not other antibody isotypes, as CD21 is present on all B cells.

The group used NMR spectroscopy to show that sCD23 can bind to both $\lg E$ and CD21 simultaneously, as their binding sites are nonoverlapping. This dual binding capacity allows SCD23 to link IgE and CD21 on the surface of B cells. How this linkage triggers $\lg E$ production is not yet known, but the process is likely to be similar to one initiated by the complement component C3d. C3d, when covalently linked to antigen, brings together the $B$ cell receptor (immunoglobulin M) and CD21. This assemblage recruits the $C D 21$-associated signaling molecule $C D 19$, which synergizes with the $B$ cell receptor to increase activation and proliferation of the cell.

Once IgE levels are high, a trimeric membrane-bound form of CD23 helps turn off $\lg E$ production, possibly by stabilizing the molecule and preventing its cleavage. The next challenge, according to senior author James McDonnell, will be to study CD23-mediated $\lg$ E regulation in animal models of allergic disease. In the meantime, these data might provide a structural basis for the development of novel inhibitors of allergic disease. JEM

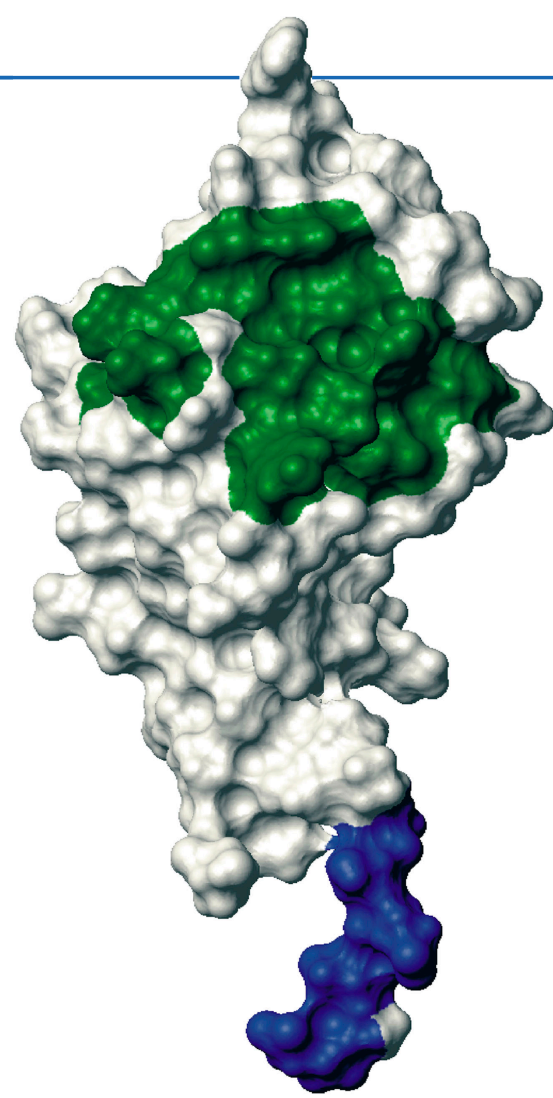

Structure of the CD23 molecule showing the binding sites for IgE (green) and CD21 (blue).

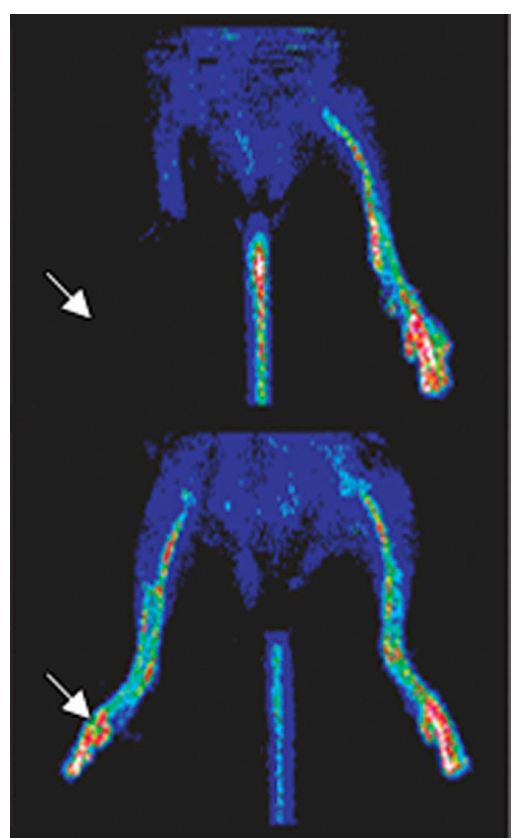

Low-dose irradiation enhances limb regeneration (bottom) unless mast cells are absent (top).

\section{Vessel-building mast cells}

Irradiation instructs mast cells to rebuild blood vessels in oxygen-deprived tissues, according to Heissig and colleagues on page 739 . Low-dose irradiation mobilized adult and precursor mast cells, which traveled to the ischemic tissue and produced the vesselbuilding growth factor VEGF (vascular endothelial growth factor).

Ionizing irradiation has been shown to promote the growth of new blood vessels through the induction of VEGF and inhibition of endothelial cell death. However, the primary source of irradiation-induced VEGF and the precise mechanism that triggers its production were not clear. Mast cells-well-known for their production of histamine in allergic responses- had been found in tissues undergoing angiogenesis. Mast cells are also capable of producing VEGF, but whether these cells play a leading or supporting role in angiogenesis had not been explored.

Heissig et al. now show that mast cells are essential for irradiation-induced vessel growth in a mouse model of ischemic limb injury. Irradiation-induced cellular damage triggers the production of the degradative enzyme matrix metalloprotease-9 (MMP-9) by largely unknown mechanisms. In this model, local irradiation of the damaged limb increased MMP-9 production in the ischemic tissue, which liberated the cytokine Kit ligand (KitL), likely from stromal cells. KitL then attracted VEGFproducing mast cells into the injured tissue.

At the same time, irradiation mobilized mast cell precursors and endothelial cell progenitors from the bone marrow, and some of these cells became incorporated into the growing vessels. Without mast cells, the vessel-building benefits of irradiation were lost. The authors thus speculate that localized irradiation might improve upon current therapies for organ regeneration, which rely on the injection of large numbers of bone marrow-derived stem cells directly into damaged organs. JEM 